\title{
Low magnetization and anisotropy in the antiferromagnetic state of undoped iron pnictides
}

\author{
E. Bascones, M.J. Calderón, and B. Valenzuela \\ Instituto de Ciencia de Materiales de Madrid, ICMM-CSIC, Cantoblanco, E-28049 Madrid (Spain) *
}

(Dated: September 15, 2021)

\begin{abstract}
We examine the magnetic phase diagram of iron pnictides using a five band model. For the intermediate values of the interaction expected to hold in the iron pnictides, we find a metallic low moment state characterized by antiparallel orbital magnetic moments. The anisotropy of the interorbital hopping amplitudes is the key to understanding this low moment state. This state accounts for the small magnetization measured in undoped iron pnictides and leads to the strong exchange anisotropy found in neutron experiments. Orbital ordering is concomitant with magnetism and produces the large $z x$ orbital weight seen at $\Gamma$ in photoemission experiments.

PACS numbers: 75.10.Jm, 75.10.Lp, 75.30.Ds
\end{abstract}

One of the common features in most iron pnictides is the appearance of unusual antiferromagnetism (AF) in the undoped compounds. In this state the magnetic moments order with momentum $\mathbf{Q}=(\pi, 0)$, namely antiferromagnetically in the $x$-direction and ferromagnetically in the $y$-direction [1]. The Curie temperatures are high $T_{N} \sim 130-200 \mathrm{~K}$, while the measured magnetic moment $m$ is small $m \sim 0.3-1.0 \mu_{B}$. A structural transition at $T_{s} \geq T_{N}$ accompanies magnetism and the system shows metallic behavior in the magnetic state. In spite of the current hot debate, the weak [2, 3] or strong coupling [4, 5] nature of magnetism is not clear yet. Abinitio calculations 2] generally report magnetic moments $m \geq 2 \mu_{B}$, much larger than experimentally measured. Proposals to explain the small magnetic moment include frustration [5], weak order [6], antiphase boundaries [7], opposite orbital magnetizations [8, 9] or the interplay between frustrated and non-frustrated bands [10].

Unexpectedly, very anisotropic nearest neighbor exchange constants $J_{y} \ll J_{x}$, with $J_{y}$ even slightly ferromagnetic, have been necessary to describe neutron scattering results [11]. Orbital ordering (OO) was proposed early on within a Kugel-Khomskii description 12 and argued to be behind both the strong anisotropy 13, 14 and the structural transition [14, 15]. Recent experiments 16, 17] have also been interpreted as manifestations of orbital ordering. In particular ARPES 16] experiments show that the Fermi surface at $\Gamma$ has a predominant $z x$ orbital component.

Here we study the magnetic phase diagram of a five band Hamiltonian at the mean field level. Different magnetic regimes, mostly coexistent with $\mathrm{OO}$, are found. At intermediate Hubbard interaction, the system is $\mathrm{AF}$ and metallic and shows two different magnetic phases. In particular, we find a low moment (LM) phase which accounts for the small magnetization measured on iron pnictides. The LM arises as a consequence of partial cancellation of antiparallel orbital magnetic moments. The anisotropy of interorbital hoppings is the key to explain this phase. To compare with neutron results, we estimate the anisotropy of the exchange interactions finding it to be strong only in the low moment state. We also show that the OO has a small contribution to the anisotropy of this state. On the other hand, the OO enhances the $z x$ orbital over the $y z$ orbital weight in the Fermi surface.

We start from the 5-band interacting Hamiltonian

$$
\begin{aligned}
& H=\sum_{i, j, \gamma, \beta, \sigma} T_{i, j}^{\gamma, \beta} c_{i, \gamma, \sigma}^{\dagger} c_{j, \beta, \sigma}+\text { h.c. }+U \sum_{j, \gamma} n_{j, \gamma, \uparrow} n_{j, \gamma, \downarrow} \\
& +U^{\prime} \sum_{j, \gamma>\beta, \sigma, \tilde{\sigma}} n_{j, \gamma, \sigma} n_{j, \beta, \tilde{\sigma}}+\frac{J}{2} \sum_{j, \gamma \neq \beta, \sigma, \tilde{\sigma}} c_{j, \gamma, \sigma}^{\dagger} c_{j, \beta, \tilde{\sigma}}^{\dagger} c_{j, \gamma, \tilde{\sigma}} c_{j, \beta, \sigma} \\
& +J^{\prime} \sum_{j, \gamma \neq \beta} c_{j, \gamma, \uparrow}^{\dagger} c_{j, \gamma, \downarrow}^{\dagger} c_{j, \beta, \downarrow} c_{j, \beta, \uparrow} .
\end{aligned}
$$

Here $i, j$ label the Fe sites in the Fe unit cell. $\gamma$ and $\beta$ refer to the five Fe-d orbitals $y z, z x, x y, 3 z^{2}-r^{2}$ and $x^{2}-y^{2}$ included in the model, and $\sigma$ to the spin. $x$ and $y$ axis are directed along the Fe bonds. The kinetic energy term includes hopping up to second neighbors with the hopping amplitudes calculated within the Slater-Koster framework [18] as detailed in Ref. [19]. Both direct Fe-Fe and indirect (via As) hoppings determine the magnitude of the hopping amplitudes. This tight binding model 19] gives good account of the band structure found in density functional theory, including the orbital content of the bands, with a reduced number of fitting parameters. The hopping amplitudes depend on the angle $\alpha$ formed by the Fe-As bonds and the Fe-plane. In the following, $\alpha=35.3^{\circ}$ corresponding to the regular tetrahedra is used, except otherwise indicated. The interacting part of the Hamiltonian includes the intraorbital $U$ and the interorbital $U^{\prime}$ interactions, as well as the Hund's coupling $J$ and pair hopping $J^{\prime}$ terms. The pair hopping interaction is written for completeness, but it does not enter in the mean-field approximation used below. Energies are given in units of $(p d \sigma)^{2} /\left|\epsilon_{d}-\epsilon_{p}\right| \sim 1 \mathrm{eV}$, with $p d \sigma$ the $\sigma$ overlap between the Fe-d and As-p orbitals and $\left|\epsilon_{d}-\epsilon_{p}\right|$ their energy difference [19]. We assume that AF takes place with $\mathbf{Q}=(\pi, \mathbf{0})$ momentum, as experimentally observed, and treat the Hamiltonian at the mean 


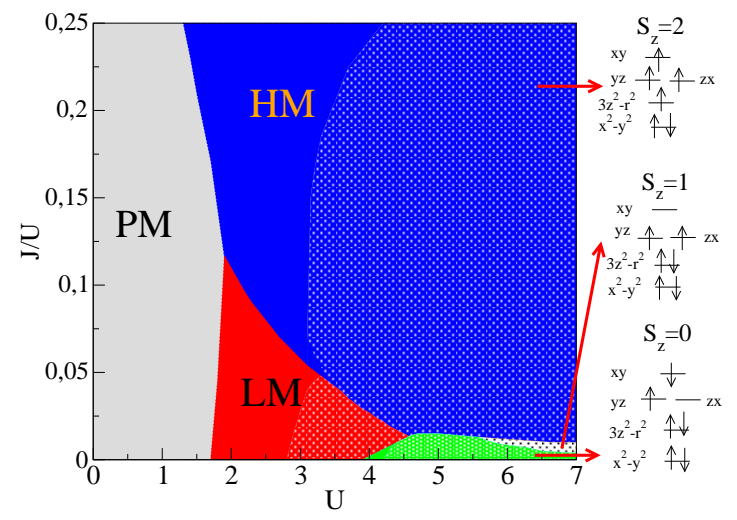

FIG. 1: (Color online) Calculated $(\pi, 0)$-mean field magnetic phase diagram of the five band Hamiltonian in Eq. 1 Patterned areas (for $U \gtrsim 3$ ) correspond to gapped states. The following color code applies: gray corresponds to the paramagnetic (PM) state; red to a low magnetic moment (LM) state showing orbital magnetizations $m_{\gamma}$ with opposite signs; blue to high magnetization (HM) with parallel $m_{\gamma}$ which corresponds at large $U$ to an $S_{z}=2$ state; white is an intermediate $S_{z}=1$ moment state; and green is the $S_{z}=0$ state. The $S_{z}=2, S_{z}=1$ and $S_{z}=0$ states are illustrated from top to bottom on the right. Magnetic moment $m$ and gap are assumed to be finite when larger than 0.001 .

field level keeping only the spin and orbital-diagonal average terms [20]

$$
n_{\gamma}=\sum_{k, \sigma}\left\langle c_{k, \gamma, \sigma}^{\dagger} c_{k, \gamma, \sigma}\right\rangle, m_{\gamma}=\sum_{k, \sigma} \sigma\left\langle c_{k+\mathbf{Q}, \gamma, \sigma}^{\dagger} c_{k, \gamma, \sigma}\right\rangle,
$$

where $k$ runs over the Fe Brillouin zone and $\sigma= \pm 1$. This corresponds to a magnetic moment $m=\sum_{\gamma} m_{\gamma}$, in units of $\mu_{B}$. We assume that the relation $U^{\prime}=U-$ $2 J$ from rotational invariance 21] holds and study the phase diagram in the $J / U$ versus $U$ space. Ferro-orbital ordering, denoted simply by $\mathrm{OO}$ in the following, and $\mathrm{AF}$ with momentum $\mathbf{Q}$ are the only symmetry breaking states allowed in our calculation. We focus on undoped systems with $n=\sum_{\gamma} n_{\gamma}=6$.

Fig. 1 displays the phase diagram with the paramagnetic (PM) state shown in gray. Different magnetic phases with momentum $\mathbf{Q}$ are represented in green, red, blue and white and correspond to states with vanishing $\left(S_{z}=0\right)$, low (LM), high (HM) and intermediate, denoted $S_{z}=1$, magnetic moment, respectively. Shaded areas (for $U \gtrsim 3$ ) correspond to insulating states with a gap at the Fermi level. The PM state, which shows no OO, survives up to a J-dependent critical value of $U$, $U_{c}(J) \sim 1.8$. The high value of $U_{c}(J)$ is not expected in a nesting scenario and suggests a strong coupling origin of AF. Moreover, the magnetic states found for intermediate values of $U \sim 2-3$ are metallic.

For large $U$ the system evolves towards the atomic limit (see the sketches in Fig. 1) with a well defined filling of orbital and spin states. For $J \sim 0.01 U$, an intermedi-
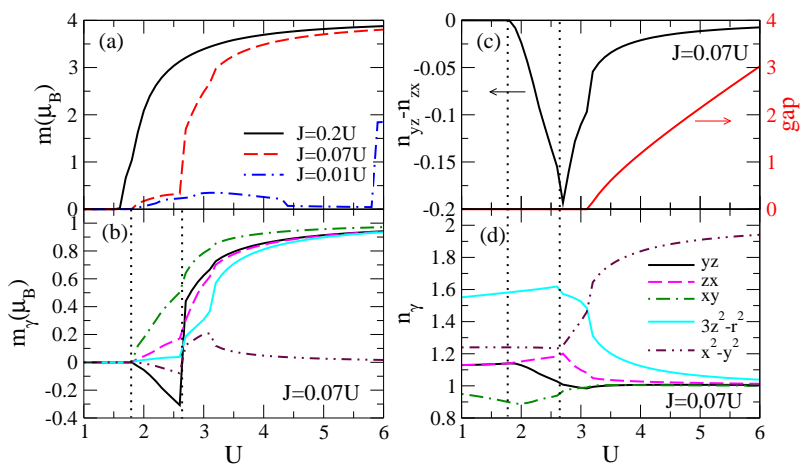

FIG. 2: (Color online) (a) Total magnetic moment for three different values of $J$ as a function of $U$. (b) Orbital magnetic moments $m_{\gamma}$, (c) OO $n_{y z}-n_{z x}$ and gap, and (d) orbital filling $n_{\gamma}$ as a function of $U$ for $J / U=0.07$. The low magnetic moment state arising for $2 \leq U \leq 2.8$ is due to the partial cancellation of opposite magnetic moments on different orbitals.

ate magnetic moment $S_{z}=1$ and insulating state appears. In this state there is no OO $\left(n_{3 z^{2}-r^{2}}, n_{x^{2}-y^{2}} \sim\right.$ $\left.2, n_{y z}, n_{z x} \sim 1\right)$ and $m_{y z}, m_{z x} \sim 1 \mu_{B}$. When $J$ increases, a spin from one of the doubly occupied states is promoted to the $x y$ orbital and we recover the $S_{z}=2$ atomic limit with $n_{x^{2}-y^{2}} \sim 2, n_{z^{2}-r^{2}}, n_{y z}, n_{z x}, n_{x y} \sim 1$ and very weak OO [see Fig. 4(b)]. In this state all the spins in the half occupied states are parallel. This tendency at large $U$ can be appreciated in Fig. 2, The HM state survives when reducing $U$ for sufficiently large values of $J / U$. At intermediate values of $U$ the system is metallic and shows OO. From Fig. 2 (a) it is apparent that, within this HM state, achieving the small magnetization $m<1 \mu_{B}$ reported experimentally is only possible by fine-tuning, as in the weak order state discussed in Ref. [6].

The $S_{z}=1$ and $S_{z}=2$ atomic states have been widely used as starting points in previous works, where the Hund's rule is assumed to hold. As shown in Fig. 1 for large $U$ and $J \simeq 0$, an $S_{z}=0$ state with a strong and positive OO $n_{y z}>>n_{z x}$ appears. In this state Hund's rule is violated. The electrons in the $x y$ and $y z$ orbitals have antiparallel spins, see sketch at the right. Most importantly, at intermediate $U$ and not too large $J / U$, we find an LM phase (with $m<1 \mu_{B}$ ) with negative $\mathrm{OO}$ $n_{y z}<n_{z x}$ in which the individual orbital magnetizations have opposite signs. In this state $m_{y z}, m_{x^{2}-y^{2}}<0$, while $m_{z x}, m_{x y}>0\left(m_{3 z^{2}-r 2} \sim 0\right)$. This is shown in Fig 2 (b) for $J / U=0.07$ within the range $1.8<U<2.7$ (between the vertical dotted lines). The low or almost vanishing total magnetic moment in these two states arises due to the partial cancellation of otherwise relatively strong magnetic moments in close to half-filling orbitals.

An AF solution with opposite orbital magnetizations, stabilized by the formation of large multipoles of the spin magnetization, was previously found in an ab-initio calculation [8] for LaOFeAs. Violation of Hund's rule in 


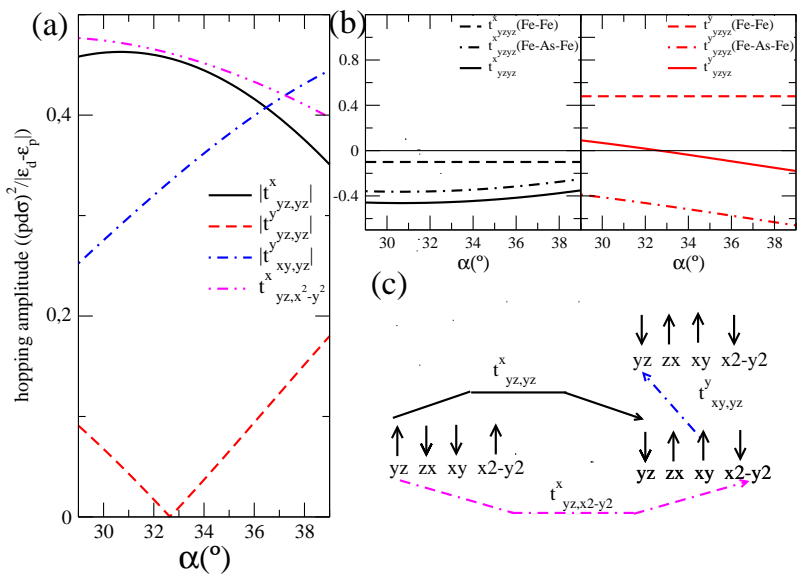

FIG. 3: (Color online) (a) Dependence on $\alpha$ of the first nearest neighbors hopping amplitudes mostly responsible for the stability of the low-magnetic moment state and the orbital ordering. For other hopping amplitudes see 19]. (b) Direct Fe-Fe and indirect (via As) contributions to $t_{y z, y z}^{x}$ and $t_{y z, y z}^{y}$ showing a strong anisotropy. (c) Sketch of the low magnetic moment state on three neighboring sites of the Fe plane and the largest hoppings involved in its stabilization.

the pnictides has also been discussed within the context of a two-orbital Heisenberg model[ 9$]$ as a consequence of large interorbital exchange $J_{\gamma, \beta}$. In the latter work, isotropic $J_{\gamma, \beta}$ were used and the columnar AF order was not found. As discussed below, in our model the stability of the $S_{z}=0$ and LM states can be understood within a strong coupling point of view by considering the anisotropy of the interorbital exchange interactions $J_{\gamma, \beta} \propto t_{\gamma, \beta}^{2}$ which override Hund's rule.

In an $\mathrm{AF}$ state with momentum $\mathbf{Q}=(\pi, 0)$, each $m_{\gamma}$ changes sign between nearest neighbors along the $x$ direction, but not along the $y$ direction. As shown in Fig. B(a), the hopping between $y z$ and $x^{2}-y^{2}$ along the $x$ direction $\left|t_{y z, x^{2}-y^{2}}^{x}\right|$ is large, while it vanishes along the $y$ direction. Therefore, parallel magnetic order of $y z$ and $x^{2}-y^{2}$ is favored as the system gains AF energy along $x$ without any cost in energy associated with the ferromagnetic ordering along $y$. On the contrary, the hopping between $y z$ and $x y$ is large along the $y$ direction $\left|t_{y z, x y}^{y}\right|$ and vanishes along $x$. This implies that parallel ordering of the $y z$ and $x y$ magnetic moments would cost exchange energy along $y$, while there would be no gain whatsoever along $x$. A configuration with opposite signs of $m_{y z}$ and $m_{x y}$ shows relative $\mathrm{AF}$ ordering of these two magnetic moments along $y$ (see sketch in Fig. 3) saving exchange interaction. From symmetry, $\left|t_{z x, x^{2}-y^{2}}^{y}\right|=\left|t_{y z, x^{2}-y^{2}}^{x}\right|$ and $\left|t_{z x, x y}^{x}\right|=\left|t_{y z, x y}^{y}\right|$ which favors antiparallel orientation between $m_{z x}$ and $m_{x^{2}-y^{2}}$ and parallel orientation between $m_{z x}$ and $m_{x y}$. Interactions involving $3 z^{2}-r^{2}$ are more frustrated, $n_{3 z^{2}-r^{2}} \sim 2$ and therefore its magnetization is small in the LM state. At large $J / U$, Hund's rule dominates and the lowest energy correspond to the HM

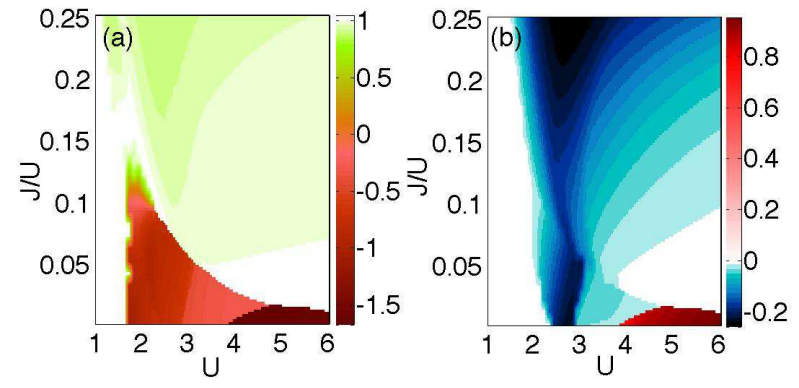

FIG. 4: (Color online) (a) Anisotropy $J_{\text {eff }}^{y} / J_{\text {eff }}^{x}$ of the magnetic exchange from a strong coupling approach and (b) orbital ordering $n_{y z}-n_{z x}$ as a function of $U$ and $J$. The anisotropy appears mostly within the $S_{z}=0$ and low moment states (green and red (LM) regions in Fig 1). The orbital ordering accompanies the magnetization within a wide range of parameters but is not correlated with the exchange anisotropy.

state.

Negative OO appears both in the LM and HM regimes [see Fig. 4(b)]. This produces a splitting of $z x$ and $y z$ bands at $\Gamma$ leading to larger weight of $z x$ at the Fermi surface (see supplementary material[22]) in agreement with ARPES experiments [16].

As discussed previously by Lee, Yin and Ku [14] based on ab-initio calculations, $\mathrm{OO}$ originates in the anisotropy of the $y z(z x)$ intraorbital first nearest neighbor hopping which favors a large (small) magnetic moment in the $y z(z x)$ orbital. Unexpectedly, $t_{y z, y z}^{x}>t_{y z, y z}^{y}\left(t_{z x, z x}^{y}>\right.$ $\left.t_{z x, z x}^{x}\right)$. This relationship is opposite to the one used in early proposals of OO in iron pnictides 12, 13]. Our tightbinding model 19 does not only reproduce Lee, Yin and $\mathrm{Ku} 14]$ results, but it also allows to understand its origin. From Fig. 3(b) it can be seen that the small value of $t_{y z, y z}^{y}$ $\left(=t_{z x, z x}^{x}\right)$ comes from the cancellation of large direct FeFe and indirect (via As) contributions with opposite sign, which add with the same sign for $t_{y z, y z}^{x}\left(=t_{z x, z x}^{y}\right)$.

In order to compare with neutron experiments[11] we make connection with a Heisenberg model and estimate the anisotropy of the exchange interactions. Within a strong coupling approach, $J_{\gamma, \beta}^{x, y} \propto\left(t_{\gamma, \beta}^{x, y}\right)^{2}$, and assuming $J_{\text {eff }}^{x, y}=\sum_{\gamma, \beta} J_{\gamma, \beta}^{x, y} \vec{s}_{\gamma, i} \vec{s}_{\beta, j} /\left(\vec{S}_{i} \vec{S}_{j}\right)$ with $s_{\gamma, j}$ and $S_{j}$ the orbital and total spin at site $i$, we have calculated the ratio $J_{\text {eff }}^{y} / J_{\text {eff }}^{x}$. The results for the anisotropy are shown as a function of $U$ and $J / U$ in Fig. (4) (a). Large anisotropy $J_{\text {eff }}^{y} \ll J_{\text {eff }}^{x}$, including negative values of $J_{\text {eff }}^{y} / J_{\text {eff }}^{x}$, is only found in the LM state. A value comparable to the experimental[11] $J_{\text {eff }}^{y} / J_{\text {eff }}^{x} \sim-0.11$ is found in the LM state close to the transition to the HM state. In the HM state, the largest calculated anisotropy corresponds to $J_{\text {eff }}^{y} / J_{\text {eff }}^{x} \sim 0.88$, very far from the experimental value. Comparing with Fig. 4 (b), it is apparent that the exchange anisotropy in Fig. 4 (a) is not strongly related to OO (which appears in most of the phase diagram), but 
to the presence of antiparallel orbital magnetizations, except for the insulating strong coupling solution $S_{z}=0$ where both effects are present. This is easily understood as the contribution of products $\vec{s}_{\gamma} \vec{s}_{\beta}$ with positive and negative sign cancel when summed up. Following a similar procedure we have also estimated the ratio of the exchange between second and first neighbors $J_{\text {eff }}^{(2)} / J_{\text {eff }}^{x}$. Its value, not shown, is around $1 / 2$ in HM state but decreases in the LM phase. This is also consistent with the experimental 11] $J_{\text {eff }}^{(2)} / J_{\text {eff }}^{x} \sim 0.38$. This suggests that second nearest neighbors are important to stabilize the HM, but not the LM state.

Several works have emphasized the influence of the Fe-As angle $\alpha$ on determining the properties of iron pnictides [23]. We have calculated the phase diagram for $\alpha=37.2^{\circ}$ (elongated tetrahedra) and for $\alpha=29.9^{\circ}$ (squashed tetrahedra as found in LaFePO). For the elongated case the phase diagram is very similar to the one discussed here for the regular tetrahedra. On the other hand, for $\alpha=29.9^{\circ}$ the low magnetic moment is less stable and reduced to a very small portion of the phase diagram. It is interesting to note that $\mathrm{AF}$ is absent in LaFePO. The worse stability of the LM state for squashed tetrahedra can be understood by looking at the angle dependence of the hopping parameters (see Fig. 3(a)): $\left|t_{y z, x y}^{y}\right|$ which helps stabilize the LM phase strongly decreases with decreasing $\alpha$.

In conclusion, we have studied the mean field magnetic phase diagram of a five orbital model for the iron pnictides. Several magnetic phases appear for different values of Hubbard and Hund interactions. OO is present for a wide range of parameters but does not seem to have a strong effect on the anisotropy of the exchange interactions in the metallic region. A metallic low magnetization state with antiparallel orbital magnetic moments is found for intermediate values of $U$. It is stabilized by the anisotropy of the interorbital hoppings, ultimately related to the symmetry of the orbitals and the tetrahedral coordination of the As atoms. This state is consistent with the measured small magnetic moment[1], the large weight of $z x$ found in the Fermi Surface around $\Gamma[16$, and the anisotropy of the exchange interactions [11.

Our results uncover antiparallel orbital magnetizations as a new source of anisotropy connecting not previously related experiments: the anisotropy measured with neutron scattering and the observed low magnetic moment. They suggest a strongly correlated origin of the magnetic state different from both the nesting scenario and the usual Heisenberg description in terms of the atomic moment and point to the need of describing correctly the individual exchange interactions between the orbital magnetic moments. They also stress the importance of including all five $\mathrm{Fe}$ d-orbitals in the description of the pnictides and question the validity of band models which mimic the band structure and Fermi surface with less orbitals as the hopping amplitudes are expected to differ considerably from the correct ones.

To the best of our knowledge, we are not aware of any technique that can make a straightforward direct measurement of the low magnetic moment state that we find. However, we expect it to show up indirectly as we have already seen in the anisotropy of the exchange interaction observed with neutron experiments.

We have benefited from conversations with $\mathrm{M}$. Daghofer, F. Yndurain, F. Mompeán, V. Scagnoli, N. Nemes, C. Prieto, N. Menéndez, and M.A. Laguna. We acknowledge funding from Ministerio de Ciencia e Innovación through Grants No. FIS 2008-00124/FIS, and No. FIS2009-08744 and Ramón y Cajal contract, and from Consejería de Educación de la Comunidad Autónoma de Madrid and CSIC through Grants No. CCG08CSIC/ESP-3518, PIE-200960I033 and PIE-200960I180.

* Electronic address: leni@icmm.csic.es,calderon@icmm.csic.es,belenv@

[1] C. de la Cruz et al., Nature 453, 899 (2008).

[2] I. Mazin et al., Phys. Rev. B 78, 085104 (2008).

[3] S. Raghu et al., Phys. Rev. B 77, 220503 (2008).

[4] Q. Si and E. Abrahams, Phys. Rev. Lett. 101, 076401 (2008).

[5] T. Yildirim, Physical Review Letters 101, 057010 (2008).

[6] E. Kaneshita et al., Phys. Rev. Lett. 103, 247202 (2009).

[7] I. Mazin and M. Johannes, Nature Physics 5, 141 (2009).

[8] F. Cricchio, O. Granas, and L. Nordstrom, arXiv:0911.1342 (2009).

[9] J. P. Rodriguez and E. H. Rezayi, Phys. Rev. Lett. 103, 097204 (2009).

[10] H. Lee et al., arXiv:0912.4024 (2009).

[11] J. Zhao et al., Nature Physics 5, 555 (2009).

[12] F. Krüger et al., Phys. Rev. B 79, 054504 (2009).

[13] R. R. P. Singh, arXiv:0903.4408 (2009).

[14] C.-C. Lee, W.-G. Yin, and W. Ku, Phys. Rev. Lett. 103, 267001 (2009).

[15] W. Lv, J. Wu, and P. Phillips, Phys. Rev. B 80, 224506 (2009).

[16] T. Shimojima et al., Phys. Rev. Lett. 104, 057002 (2010).

[17] T.-M. Chuang et al., Science 327, 181 (2010); S. H. Lee et al., arXiv:0912.3205 (2009); S. Nandi et al., Phys. Rev. Lett. 104, 057006 (2010); R. M. Fernandes et al., arXiv:0911.3084 (2009); J. G. Analytis et al., arXiv:0911.3878 (2009).

[18] J. Slater and G. Koster, Phys. Rev. 94, 1498 (1954).

[19] M. J. Calderón, B. Valenzuela, and E. Bascones, Phys. Rev. B 80, 094531 (2009).

[20] R. Yu et al., Phys. Rev. B 79, 104510 (2009); M. Daghofer et al., Phys. Rev. B 81, 014511 (2010); S. Zhou and Z. Wang, arXiv:0910.2707 (2009).

[21] C. Castellani, C. R. Natoli, and J. Ranninger, Phys. Rev. B 18, 4945 (1978).

[22] See http://link.aps.org/supplemental/10.1103/ PhysRevLett.104.227201 for a plot of the $\mathrm{zx}$ and yz contributions to the band structure around $\Gamma$.

[23] C. H. Lee et al., J. Phys. Soc. Jpn. 77, 083704 (2008); K. Kuroki et al., Phys. Rev. B 79, 224511 (2009). 